\title{
Winter Map Inverses
}

\section{Thomas B. Gregory}

Department of Mathematics, The Ohio State University at Mansfield, Mansfield, USA

Email: gregory.6@osu.edu

Received 24 April 2014; revised 20 May 2014; accepted 3 June 2014

Copyright (C) 2014 by author and Scientific Research Publishing Inc.

This work is licensed under the Creative Commons Attribution International License (CC BY).

http://creativecommons.org/licenses/by/4.0/

\section{(c) (i) Open Access}

\section{Abstract}

We demonstrate the functional inverse of a Winter map, which is an analog of the exponential map, for Lie algebras over fields of prime characteristic.

\section{Keywords}

Prime-Characteristic Lie Algebras, Prime-Characteristic Lie Groups

"Historically,” note Strade and Farnsteiner in [1], "Lie algebras emerged from the study of Lie groups.” In Section 1.1 of [1], they give a simple example of the close connection between Lie algebras and Lie groups. In prime characteristic, David Winter [2] has defined maps which mimic the zero-characteristic exponential maps. See also Lemma 1.2 of [3]. In this paper, we focus on the following "Winter maps": if $x$ is an element of a characteristic- $p$ Lie algebra $L$ such that $\left(\operatorname{ad}_{L} x\right)^{p}=0$, we set

$$
\xi\left(\operatorname{ad}_{L} x\right)=I+\operatorname{ad}_{L} x+\frac{\left(\operatorname{ad}_{L} x\right)^{2}}{2 !}+\frac{\left(\operatorname{ad}_{L} x\right)^{3}}{3 !}+\cdots+\frac{\left(\operatorname{ad}_{L} x\right)^{p-1}}{(p-1) !}
$$

where $I$ is the identity transformation of $L$. Such ad-nilpotent elements of degree less than $p$ do exist in some graded Lie algebras, as can be seen from Lemma 2.3 and Proposition 2.7 of Chapter 4 of [1], as well as from Lemma 1 of [4]; of course, it is well known that non-zero-root vectors of simple classical-type Lie algebras are ad-nilpotent of degree less than or equal to four.

We will show here that for $x \in L$ such that $\left(\operatorname{ad}_{L} x\right)^{p}=0$, the inverse of $\xi\left(\operatorname{ad}_{L} x\right)$ as a linear transformation of $L$ is $\xi\left(\operatorname{ad}_{L}(-x)\right)$, so that such transformations generate a group $G$ of linear transformations of $L$. We will also show that $\lambda\left(\xi\left(\operatorname{ad}_{L} x\right)\right)=\operatorname{ad}_{L} x$, where, for $g$ a linear transformation of $L$, and $I$ as above, we define

$$
\lambda(g)=(g-I)-\frac{(g-I)^{2}}{2}+\frac{(g-I)^{3}}{3}-\cdots \frac{(g-I)^{p-1}}{(p-1) !}
$$

Thus, like $\ln (x)$ and $\exp (x), \lambda$ is, in a sense, the functional inverse of $\xi$. 
Lemma 1 If $x$ and $c$ are elements of $L$ such that $\left(\operatorname{ad}_{L} x\right)^{p}=0$, and $\left(\operatorname{ad}_{L} c\right)^{p}=0$, then

$$
\begin{aligned}
\xi(x) \xi(c) & =\sum_{i=0}^{p-1} \sum_{j=0}^{i} \frac{\left(\operatorname{ad}_{L} x\right)^{j}(\operatorname{ad} c)^{i-j}}{j !(i-j) !} \\
& +\sum_{i=p}^{2 p-2} \sum_{j=i-(p-1)}^{p-1} \frac{\left(\operatorname{ad}_{L} x\right)^{j}(\operatorname{ad} c)^{i-j}}{j !(i-j) !}
\end{aligned}
$$

Proof. We group terms with respect to total degree in ad ${ }_{L} X$ and $\operatorname{ad}{ }_{L} C \quad \square$

Lemma 2 Let $a, b \in F$, and suppose that $x$ is an element of $L$ such that $\left(\operatorname{ad}_{L} x\right)^{p}=0$. then

$$
\xi\left((a) \operatorname{ad}_{L} x\right) \xi\left((b) \operatorname{ad}_{L} x\right)=\xi\left((a+b) \operatorname{ad}_{L} x\right) .
$$

Proof. We have by Lemma 1 that $\xi\left((a) \operatorname{ad}_{L} x\right) \xi\left((b) \operatorname{ad}_{L} x\right)$ equals

$$
\sum_{i=0}^{p-1} \sum_{j=0}^{i} \frac{a^{j} b^{i-j}\left(\operatorname{ad}_{L} x\right)^{i}}{j !(i-j) !}+0
$$

which we can write in terms of binomial coefficients as

$$
\sum_{i=0}^{p-2} \frac{\left(\operatorname{ad}_{L} x\right)^{i}}{i !} \sum_{j=0}^{i}\left(\begin{array}{l}
i \\
j
\end{array}\right) a^{j} b^{i-j}
$$

By the Binomial Theorem, the above expression is equal to

$$
\sum_{i=0}^{p-1} \frac{\left(\operatorname{ad}_{L} x\right)^{i}}{i !}(a+b)^{i}
$$

which we can rewrite as

$$
\sum_{i=0}^{p-1} \frac{\left(\operatorname{ad}_{L}(a+b) x\right)^{i}}{i !}
$$

and recognize as $\xi\left((a+b)\right.$ ad $\left._{L} x\right)$.

Lemma 3 For any integer $n \geqq 2$ and any integer $j, 0<j<n$, we have

$$
\sum_{k=0}^{n}(-1)^{k}\left(\begin{array}{l}
n \\
k
\end{array}\right) k^{j}=0
$$

Proof. We proceed by induction on $n$ and $j$. When $n=2$, we must have $j=1$, and we have $0-2+2=0$. For any $n>2$, when $j=1$, we have

$$
\begin{aligned}
\sum_{k=0}^{n}(-1)^{k}\left(\begin{array}{l}
n \\
k
\end{array}\right) k^{1} & =\sum_{k=0}^{n-1}(-1)^{k+1}\left(\begin{array}{c}
n \\
k+1
\end{array}\right)(k+1) \\
& =\sum_{k=0}^{n-1}(-1)^{k+1} \frac{n !}{(k+1) !(n-(k+1)) !}(k+1) \\
& =(-n) \sum_{k=0}^{n-1}(-1)^{k} \frac{(n-1) !}{k !((n-1)-k) !} \\
& =(-n) \sum_{k=0}^{n-1}(-1)^{k}\left(\begin{array}{c}
n-1 \\
k
\end{array}\right) \\
& =(-n) \cdot(1-1)^{n-1}=0 .
\end{aligned}
$$

Now, for any $n \geqq 3$ and any positive integer $j$ less than $n$, suppose that $\sum_{k=1}^{n}(-1)^{k}\left(\begin{array}{l}n \\ k\end{array}\right) k^{i}=0$ for all positive $i$ less than $j$. Then we have 


$$
\begin{aligned}
\sum_{k=0}^{n}(-1)^{k}\left(\begin{array}{l}
n \\
k
\end{array}\right) k^{j} & =\sum_{k=1}^{n}(-1)^{k} \frac{n !}{k !(n-k) !} k^{j}=(-n) \sum_{k=1}^{n}(-1)^{k-1} \frac{(n-1) !}{(k-1) !(n-k) !} k^{j-1} \\
= & (-n) \sum_{k=1}^{n}(-1)^{k-1} \frac{(n-1) !}{(k-1) !((n-1)-(k-1)) !} k^{j-1} \\
& =(-n) \sum_{k=1}^{n}(-1)^{k-1}\left(\begin{array}{c}
n-1 \\
k-1
\end{array}\right) k^{j-1} \\
& =(-n) \sum_{k=0}^{n-1}(-1)^{k}\left(\begin{array}{c}
n-1 \\
k
\end{array}\right)(k+1)^{j-1} \\
& =(-n)\left\{\sum_{k=1}^{n-1}(-1)^{k}\left(\begin{array}{c}
n-1 \\
k
\end{array}\right) \sum_{i=1}^{j-1}\left(\begin{array}{c}
j-1 \\
i
\end{array}\right) k^{i}+\sum_{k=0}^{n-1}(-1)^{k}\left(\begin{array}{c}
n-1 \\
k
\end{array}\right)\right\} \\
& =(-n) \sum_{i=1}^{j-1}\left(\begin{array}{c}
j-1 \\
i
\end{array}\right) \sum_{k=1}^{n-1}(-1)^{k}\left(\begin{array}{c}
n-1 \\
k
\end{array}\right) k^{i}+0=(-n) \sum_{i=1}^{j-1}\left(\begin{array}{c}
j-1 \\
i
\end{array}\right) \cdot 0=0
\end{aligned}
$$

by induction, and the fact that $\sum_{k=0}^{n}(-1)^{k}\left(\begin{array}{l}n \\ k\end{array}\right)=(1-1)^{n}=0^{n}=0 \quad$ (the “ $j=0$ case”).

Lemma 4 Let $x$ be an element of $L$ such that $\left(\operatorname{ad}_{L} x\right)^{p}=0$. Define

$$
\delta\left(\operatorname{ad}_{L} x\right)=\sum_{i=0}^{p-2} \frac{\left(\operatorname{ad}_{L} x\right)^{i+1}}{(i+1) !}
$$

Then for any positive integer $n$ less than $p$,

$$
\left(\delta\left(\operatorname{ad}_{L} x\right)\right)^{n}=\sum_{t=0}^{p-n-1} \frac{\left(\operatorname{ad}_{L} x\right)^{t+n}}{(t+n) !}\left(\sum_{j=0}^{n-1}\left(\begin{array}{l}
n \\
j
\end{array}\right)(-1)^{j}(n-j)^{t+n}\right)
$$

Proof. We proceed by induction on $n$. Since when $n=1$, (3) is just (2), the initial step of the induction proof is established. Suppose (3) is true for $n=k \geqq 1$. Then $\left(\delta\left(\operatorname{ad}_{L} x\right)\right)^{k+1}$ equals

$$
\left(\sum_{s=0}^{p-k-1} \frac{\left(\mathrm{ad}_{L} x\right)^{s+k}}{(s+k) !} \sum_{j=0}^{k-1}\left(\begin{array}{l}
k \\
j
\end{array}\right)(-1)^{j}(k-j)^{s+k}\right)\left(\sum_{i=0}^{p-2} \frac{\left(\mathrm{ad}_{L} x\right)^{i+1}}{(i+1) !}\right)
$$

We group terms with respect to total degree $\left(t+k+1\right.$, in this case) in $\operatorname{ad}_{L} X$ and get that

$$
\left(\delta\left(\operatorname{ad}_{L} x\right)\right)^{k+1}=\sum_{t=0}^{p-(k+1)-1} \sum_{r=0}^{t} \frac{\left(\operatorname{ad}_{L} x\right)^{r+k}\left(\operatorname{ad}_{L} x\right)^{t-r+1}}{(r+k) !(t-r+1) !}\left(\sum_{j=0}^{k-1}\left(\begin{array}{c}
k \\
j
\end{array}\right)(-1)^{j}(k-j)^{r+k}\right) .
$$

Rewriting the above expression using another binomial coefficient, we get that $\left(\delta\left(\operatorname{ad}_{L} x\right)\right)^{k+1}$ equals

$$
\sum_{t=0}^{p-(k+1)-1} \frac{\left(\mathrm{ad}_{L} x\right)^{t+k+1}}{(t+k+1) !} \sum_{r=0}^{t} \sum_{j=0}^{k-1}(-1)^{j}\left(\begin{array}{c}
k \\
j
\end{array}\right)(k-j)^{r+k}\left(\begin{array}{c}
t+k+1 \\
r+k
\end{array}\right) .
$$

We change the order of summation to get

$$
\left(\delta\left(\operatorname{ad}_{L} x\right)\right)^{k+1}=\sum_{t=0}^{p-(k+1)-1} \frac{\left(\operatorname{ad}_{L} x\right)^{t+k+1}}{(t+k+1) !} \sum_{j=0}^{k-1}(-1)^{j}\left(\begin{array}{c}
k \\
j
\end{array}\right) \sum_{r=0}^{t}\left(\begin{array}{c}
t+k+1 \\
r+k
\end{array}\right)(k-j)^{r+k} .
$$

We replace the index of summation $r$ by $r-k$ to get

$$
\left(\delta\left(\operatorname{ad}_{L} x\right)\right)^{k+1}=\sum_{t=0}^{p-(k+1)-1} \frac{\left(\operatorname{ad}_{L} x\right)^{t+k+1}}{(t+k+1) !} \sum_{j=0}^{k-1}(-1)^{j}\left(\begin{array}{c}
k \\
j
\end{array}\right) \sum_{r=k}^{t+k}\left(\begin{array}{c}
t+k+1 \\
r
\end{array}\right)(k-j)^{r} .
$$


Adding and subtracting terms, we get

$$
\begin{aligned}
\left(\delta\left(\operatorname{ad}_{L} x\right)\right)^{k+1}= & \sum_{t=0}^{p-(k+1)-1} \frac{\left(\operatorname{ad}_{L} x\right)^{t+k+1}}{(t+k+1) !} \sum_{j=0}^{k-1}(-1)^{j}\left(\begin{array}{c}
k \\
j
\end{array}\right)\left\{\sum_{r=0}^{t+k+1}\left(\begin{array}{c}
t+k+1 \\
r
\end{array}\right)(k-j)^{r}\right. \\
& \left.-\sum_{r=0}^{k-1}\left(\begin{array}{c}
t+k+1 \\
r
\end{array}\right)(k-j)^{r}-\sum_{r=t+k+1}^{t+k+1}\left(\begin{array}{c}
t+k+1 \\
r
\end{array}\right)(k-j)^{r}\right\}
\end{aligned}
$$

Setting $q=k-j$, we see, as in the proof of Lemma 3, that when $r \geq 1$,

$$
\sum_{j=0}^{k-1}(-1)^{j}\left(\begin{array}{c}
k \\
j
\end{array}\right)(k-j)^{r}=(-1)^{k} k \sum_{q=1}^{k}(-1)^{q}\left(\begin{array}{c}
k-1 \\
q-1
\end{array}\right) q^{r-1}=0
$$

by that same Lemma 3. Thus,

$$
\begin{aligned}
& \sum_{j=0}^{k-1}(-1)^{j}\left(\begin{array}{c}
k \\
j
\end{array}\right) \sum_{r=0}^{k-1}\left(\begin{array}{c}
t+k+1 \\
r
\end{array}\right)(k-j)^{r} \\
& =\sum_{r=0}^{k-1}\left(\begin{array}{c}
t+k+1 \\
r
\end{array}\right) \sum_{j=0}^{k-1}(-1)^{j}\left(\begin{array}{l}
k \\
j
\end{array}\right)(k-j)^{r}=\sum_{r=0}^{k-1}\left(\begin{array}{c}
t+k+1 \\
r
\end{array}\right) \cdot 0=0,
\end{aligned}
$$

so from the Binomial Theorem, we get that $\left(\delta\left(\operatorname{ad}_{L} x\right)\right)^{k+1}$ equals

$$
\sum_{t=0}^{p-(k+1)-1} \frac{\left(\mathrm{ad}_{L} x\right)^{t+k+1}}{(t+k+1) !} \sum_{j=0}^{k-1}(-1)^{j}\left(\begin{array}{c}
k \\
j
\end{array}\right)\left\{(k-j+1)^{t+k+1}-1-(k-j)^{t+k+1}\right\} .
$$

We now distribute to get that $\left(\delta\left(\operatorname{ad}_{L} x\right)\right)^{k+1}$ equals

$$
\sum_{t=0}^{p-(k+1)-1} \frac{\left(\operatorname{ad}_{L} x\right)^{t+k+1}}{(t+k+1) !}\left\{\sum_{j=0}^{k-1}\left(\begin{array}{l}
k \\
j
\end{array}\right)(-1)^{j}(k-j+1)^{t+k+1}+(-1)^{k}-\sum_{j=0}^{k-1}\left(\begin{array}{l}
k \\
j
\end{array}\right)(-1)^{j}(k-j)^{t+k+1}\right\} .
$$

We replace the latter index of summation $j$ by $j-1$ to get that $\left(\delta\left(\operatorname{ad}_{L} x\right)\right)^{k+1}$ equals

$$
\sum_{t=0}^{p-(k+1)-1} \frac{\left(\mathrm{ad}_{L} x\right)^{t+k+1}}{(t+k+1) !}\left\{\sum_{j=0}^{k-1}\left(\begin{array}{c}
k \\
j
\end{array}\right)(-1)^{j}(k-j+1)^{t+k+1}-\sum_{j=1}^{k}\left(\begin{array}{c}
k \\
j-1
\end{array}\right)(-1)^{j-1}(k+1-j)^{t+k+1}+(-1)^{k}\right\} .
$$

We change the order of summation and factor to get that $\left(\delta\left(\operatorname{ad}_{L} x\right)\right)^{k+1}$ equals

$$
\sum_{t=0}^{p-(k+1)-1} \frac{\left(\mathrm{ad}_{L} \mathrm{X}\right)^{t+k+1}}{(t+k+1) !}\left\{(k+1)^{t+k+1}+\sum_{j=1}^{k-1}\left\{\left(\begin{array}{c}
k \\
j
\end{array}\right)(-1)^{j}+\left(\begin{array}{c}
k \\
j-1
\end{array}\right)(-1)^{j}\right\}(k+1-j)^{t+k+1}-\left(\begin{array}{c}
k \\
k-1
\end{array}\right)(-1)^{k-1}-(-1)^{k-1}\right\} .
$$

By binomial arithmetic $\left(\delta\left(\operatorname{ad}_{L} x\right)\right)^{k+1}$ equals

$$
\sum_{t=0}^{p-(k+1)-1} \frac{\left(\mathrm{ad}_{L} x\right)^{t+k+1}}{(t+k+1) !}\left\{(k+1)^{t+k+1}+\sum_{j=1}^{k-1}\left(\begin{array}{c}
k+1 \\
j
\end{array}\right)(-1)^{j}(k+1-j)^{t+k+1}-(k+1)(-1)^{k-1}\right\} .
$$

The above displayed formula is just (3) for $n=k+1$; i.e., $\left(\delta\left(\operatorname{ad}_{L} x\right)\right)^{k+1}$ equals

$$
\sum_{t=0}^{p-(k+1)-1} \frac{\left(\operatorname{ad}_{L} x\right)^{t+k+1}}{(t+k+1) !}\left\{\sum_{j=0}^{k}(-1)^{j}\left(\begin{array}{c}
k+1 \\
j
\end{array}\right)(k+1-j)^{t+k+1}\right\} .
$$

Thus, the induction step is complete. $\square$

Theorem The linear transformation $\xi\left(\operatorname{ad}_{L} x\right)$ of $L$ has $\xi\left(\operatorname{ad}_{L}(-x)\right)$ as its inverse, whereas the map $\xi$ of ad $L$ to the group of non-singular linear transformations of $L$ has $\lambda$ as its inverse, in the sense that 
(a). $\xi\left(\operatorname{ad}_{L} x\right) \xi\left(\operatorname{ad}_{L}(-x)\right)=I$, and

(b). $\lambda\left(\xi\left(\operatorname{ad}_{L} x\right)\right)=\operatorname{ad}_{L} x$.

Proof. (a) If, in Lemma 2, we let $a=1$ and $b=-1$, we see that (a) is true.

(b) Since $\xi\left(\operatorname{ad}_{L} x\right)-I$ equals the $\delta\left(\operatorname{ad}_{L} x\right)$ of Lemma 4 , we have that $\lambda\left(\xi\left(\operatorname{ad}_{L} x\right)\right)$ equals

$$
\left(\delta\left(\operatorname{ad}_{L} x\right)\right)-\frac{\left(\delta\left(\operatorname{ad}_{L} x\right)\right)^{2}}{2}+\frac{\left(\delta\left(\operatorname{ad}_{L} x\right)\right)^{3}}{3}-\cdots-\frac{\left(\delta\left(\operatorname{ad}_{L} x\right)\right)^{p-1}}{p-1}
$$

which, by Lemma 4 equals

$$
\sum_{n=1}^{p-1} \frac{(-1)^{n+1}}{n}\left\{\sum_{t=0}^{p-n-1} \frac{\left(\operatorname{ad}_{L} x\right)^{t+n}}{(t+n) !}\left(\sum_{j=0}^{n-1}\left(\begin{array}{l}
n \\
j
\end{array}\right)(-1)^{j}(n-j)^{t+n}\right)\right\}
$$

We replace the index $t$ by $t-n$ to get that

$$
\lambda\left(\xi\left(\operatorname{ad}_{L} x\right)\right)=\sum_{n=1}^{p-1} \frac{(-1)^{n+1}}{n}\left\{\sum_{t=n}^{p-1} \frac{\left(\operatorname{ad}_{L} x\right)^{t}}{t !}\left(\sum_{j=0}^{n-1}\left(\begin{array}{l}
n \\
j
\end{array}\right)(-1)^{j}(n-j)^{t}\right)\right\}
$$

We change the order of summation to get that

$$
\lambda\left(\xi\left(\operatorname{ad}_{L} x\right)\right)=\sum_{t=1}^{p-1} \frac{\left(\operatorname{ad}_{L} x\right)^{t}}{t !} \sum_{n=1}^{t} \frac{(-1)^{n+1}}{n}\left\{\sum_{j=0}^{n-1}\left(\begin{array}{l}
n \\
j
\end{array}\right)(-1)^{j}(n-j)^{t}\right\}
$$

We replace the index $j$ by $n-j$ to get that

$$
\lambda\left(\xi\left(\operatorname{ad}_{L} x\right)\right)=\sum_{t=1}^{p-1} \frac{\left(\operatorname{ad}_{L} x\right)^{t}}{t !} \sum_{n=1}^{t} \frac{(-1)^{n+1}}{n}\left\{\sum_{j=1}^{n}\left(\begin{array}{l}
n \\
j
\end{array}\right)(-1)^{n-j} j^{t}\right\}
$$

We cancel an $n$ and a $j$ and combine the -1 factors to get that

$$
\lambda\left(\xi\left(\operatorname{ad}_{L} x\right)\right)=\sum_{t=1}^{p-1} \frac{\left(\operatorname{ad}_{L} x\right)^{t}}{t !} \sum_{n=1}^{t}\left\{\sum_{j=1}^{n}\left(\begin{array}{c}
n-1 \\
j-1
\end{array}\right)(-1)^{j-1} j^{t-1}\right\}
$$

We replace the index $n$ by $n+1$ and we replace the index $j$ by $j+1$, and we get that

$$
\lambda\left(\xi\left(\operatorname{ad}_{L} x\right)\right)=\sum_{t=1}^{p-1} \frac{\left(\operatorname{ad}_{L} x\right)^{t}}{t !} \sum_{n=0}^{t-1}\left\{\sum_{j=0}^{n}\left(\begin{array}{c}
n \\
j
\end{array}\right)(-1)^{j}(j+1)^{t-1}\right\}
$$

We change the order of summation to get that

$$
\lambda\left(\xi\left(\operatorname{ad}_{L} x\right)\right)=\sum_{t=1}^{p-1} \frac{\left(\operatorname{ad}_{L} x\right)^{t}}{t !} \sum_{j=0}^{t-1}(-1)^{j}(j+1)^{t-1} \sum_{n=j}^{t-1}\left(\begin{array}{l}
n \\
j
\end{array}\right)
$$

We now appeal to a little more binomial arithmetic to observe that since $\left(\begin{array}{l}j \\ j\end{array}\right)=\left(\begin{array}{c}j+1 \\ j+1\end{array}\right)$ and $\left(\begin{array}{l}t \\ j\end{array}\right)+\left(\begin{array}{c}t \\ j+1\end{array}\right)=\left(\begin{array}{c}t+1 \\ j+1\end{array}\right)$, it follows by induction that

$$
\sum_{n=j}^{t-1}\left(\begin{array}{l}
n \\
j
\end{array}\right)=\left(\begin{array}{c}
t \\
j+1
\end{array}\right)
$$

from which we obtain that

$$
\lambda\left(\xi\left(\operatorname{ad}_{L} x\right)\right)=\sum_{t=1}^{p-1} \frac{\left(\operatorname{ad}_{L} x\right)^{t}}{t !} \sum_{j=0}^{t-1}(-1)^{j}(j+1)^{t-1}\left(\begin{array}{c}
t \\
j+1
\end{array}\right)
$$


We replace the index $j$ by $j-1$ to get that

$$
\lambda\left(\xi\left(\operatorname{ad}_{L} x\right)\right)=\sum_{t=1}^{p-1} \frac{\left(\operatorname{ad}_{L} x\right)^{t}}{t !} \sum_{j=1}^{t}(-1)^{j-1}\left(\begin{array}{c}
t \\
j
\end{array}\right) j^{t-1}
$$

Finally, we use Lemma 3 to see that we are left with $\operatorname{ad}_{L} X \quad \square$

\section{References}

[1] Strade, H. and Farnsteiner, R. (1988) Modular Lie Algebras and Their Representations. Pure and Applied Mathematics, 116, Dekker, New York.

[2] Winter, D.J. (1969) On the Toral Structure of Lie p-Algebras. Acta Mathematica, 123, 70-81.

[3] Weisfeiler, B.J. and Kac, V.G. (1971) Exponentials in Lie Algebras of Characteristic p. Mathematics of the USSR-Izvestiya, 5, 777-803.

[4] Gregory, T.B. (1990) A Characterization of the General Lie Algebras of Cartan Type. In: Benkart and Osborne, Eds., Lie Algebras and Related Topics, 22 May-1 June 1988, American Mathematical Society, Madison, 75-78. 
Scientific Research Publishing (SCIRP) is one of the largest Open Access journal publishers. It is currently publishing more than 200 open access, online, peer-reviewed journals covering a wide range of academic disciplines. SCIRP serves the worldwide academic communities and contributes to the progress and application of science with its publication.

Other selected journals from SCIRP are listed as below. Submit your manuscript to us via either submit@scirp.org or Online Submission Portal.
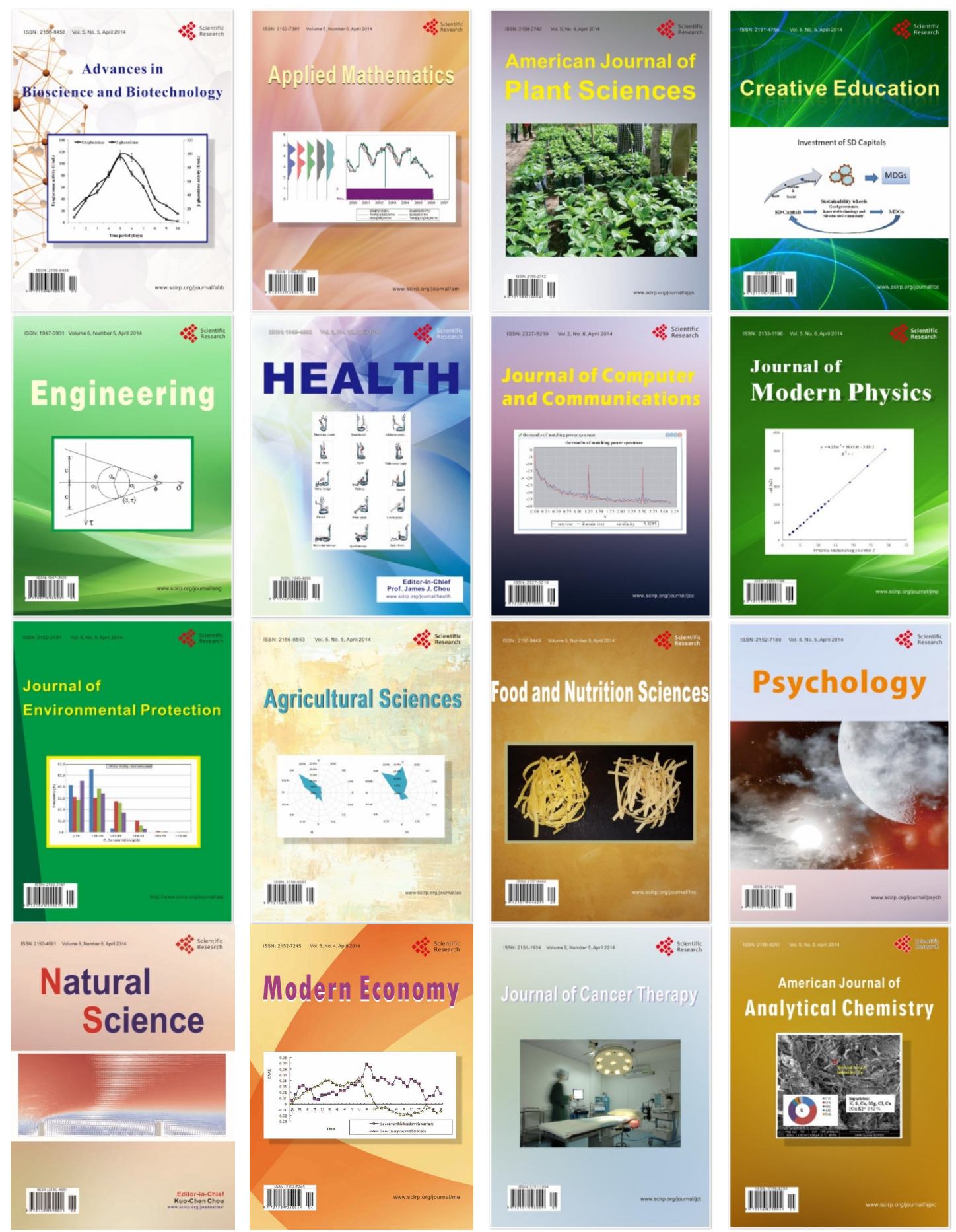